\title{
Editorial for special issue on SDN
}

\author{
S. V. Raghavan ${ }^{1,2}$
}

Published online: 22 April 2020

(C) CSI Publications 2020

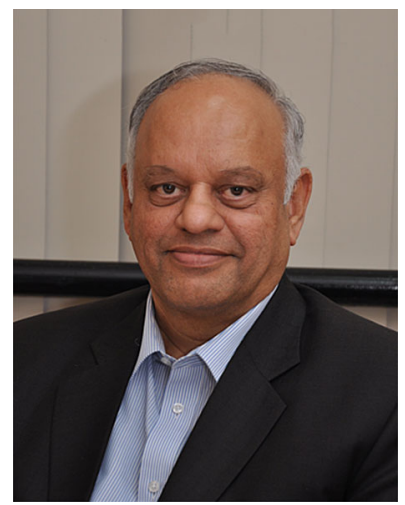

It is my privilege to introduce you to the March 2020 issue through this editorial. What started as "networking" several decades ago, has now evolved as Integrated Digital Infrastructure with associated controls that brings in fantastic flexibility through Software Defined Networking. The March 2020 issue is a transcript of what happened in the ICSDN 2019 conference in August 2019. Almost every talk is covered vividly by the team at Anna University, led by Professor Krishna Sivalingam, IIT Madras, Professor Arul Siromoney, Anna University, and Professor Anita, Anna University. Towards the end of the issue, I have added an invited article to show the direction forward.

S. V. Raghavan

sv.raghavan@gov.in

Chief Editor, CSI Transactions on ICT, Chennai 600036, India

2 Government of India, New Delhi, India

\section{The beginning}

The idea of networking started in 1961 and was established as a working artifact eight years later in 1969. The rest of the twentieth century saw the perfection of the process of packet based digital communication. The manifestation of every network that was built led to the common perception that networks are hierarchical. Partly true, of course. Well, I might very well concede, it is largely true, perhaps. Number of networks were established, mostly for research and education. Indian education and research network (of which the author was a founder) was established in 1986. Emails were flowing all over the world. Close to the end of the century, Internet became commercial. Electronic commerce was born. During these fifty years, the researchers and designers understood the value of speed, timeliness (latency) of actions, and sharing of (any) resource.

\section{Around 60 years of age}

Turn of the century and through the first decade of twentyfirst century, saw phenomenal rise of the communication medium called fiber optic cable. In the last 19 years the speed multiplied million-fold. Semiconductor technology and integrated circuit fabrication made every active electronic equipment smaller and faster, exponentially. Economies of scale was established. Designers conquered speed, latency, and sharing thereby enabling flexibility in the hands of the user. Many hierarchical networks of the previous century became coalesced into a single integrated infrastructure. Network became the lifeline of Banks, Securities, Finance, and Insurance industry. Amazon and eBay became a life partner for everyone. YouTube, 
Facebook, Twitter, WhatsApp became a way of life. A generation was born into this information age. Guarantees, Reliability, Resilience, and Agility became the key requirement in design. Extreme automation was imminent. Software Defined Networking (SDN) was born. Virtualization of every networking function, making a hierarchical network on the fly, and having fine controls through SDN is becoming the norm.

\section{First half of this century}

The first two decades saw extensive use and experimentation with open hardware, open software, and SDN. Emerging 5G and IoT and their deployment for education and health is the pursuit of every researcher, developer, investor, operator. Use of Artificial Intelligence and Machine Learning to build an anti-fragile network out of an Integrated Digital Infrastructure (IDI) that can recover from failure on its own and continue its operation, is the dream of a designer today. World is moving towards highly integrated and highly controllable IDI with a high level of granularity of observation, to keep the errors under control. Of course, there are challenges in several domains of discourse that surround IDI and SDN; they are, policy, regulation, legal framework, law enforcement, (cyber) security, privacy, and safety. Speeds are increasing, time available to respond is decreasing, ability to observe every little thing in cyber space is improving (Big Data), ability to comprehend and respond is depending on AI and ML. It is no longer a single musical instrument in an evening concert. SDN controlled IDI is an orchestra. Composing music and conducting the orchestra will mature in the next three decades.

\section{How do we prepare our nation?}

Single most important step is to develop manpower to conquer the different dimensions of SDN. They are VNF (Virtual Network Function), NFV (Network Function virtualisation), 5G, Business Models, Dynamic Service Launch, Agility in Data Analytics and response. The methodology of preparing the workforce could be establishment of several public-funded laboratories that work in close coordination across the SDN dimensions. Focus should be on producing Creative Designers who understand everything from policy plane to action plane is required. Of course, a large complimentary workforce to operate the IDIs and SDN derived artifacts is a must; perhaps in the ratio 20:80. The studies in engineering institutions (concentrates only on technology), management institutions (concentrates only on business models), and universities (concentrates on science, psychology, evolutionary behavior, etc.) have to be brought together in a creative way through the public-funded laboratories.

\section{What next?}

The Telecommunication Service Provider of today will morph to be the Digital Service Provider of tomorrow. Perhaps the State may take on the responsibility to provide the IDI and the fine controls of SDN associated with a business opportunity be provided by the DSPs. After all, in a rapidly changing opportunity space such as cyber environment, from National Security stand-point complimentary roles as suggested above makes sense. Perhaps, understanding the issues related to the operational aspects and security associated with a large complex network is the key. We close the issue with a paper that focuses on the operational aspects of the emerging complex networks of tomorrow.

Happy Reading... 